Annals of Pure and Applied Mathematics

Vol. 14, No. 1, 2017, 189-198

ISSN: 2279-087X (P), 2279-0888(online)

Published on 17 August 2017

www.researchmathsci.org

Annals of

Pure and Applied

Mathematics

\title{
Fixed Point Theorem for Occasionally Weakly Compatible Mappings in Modified Intuitionistic Fuzzy Metric Space
}

\author{
Syed Shahnawaz Ali, Jainendra Jain ${ }^{2}$, P.L. Sanodiya ${ }^{3}$ and Shilpi Jain ${ }^{4}$ \\ ${ }^{1}$ Department of Basic Sciences, Corporate Institute of Science \& Technology \\ Hataikheda, Near Patel Nagar, Raisen Road, Bhopal, M.P., India. \\ E-mail: drsyedshahnawazali@gmail.com \\ ${ }^{2}$ Department of Mathematics, Government Engineering College, Darampura Jagdalpur \\ Chhattisgarh, India. E-mail: ij.28481@gmail.com \\ ${ }^{3}$ Department of Mathematics, Institute for Excellence in Higher Education \\ Kaliyasot Dam, Kolar Road, Bhopal, M.P., India. E-mail:sanodiyapl@gmail.com \\ ${ }^{4}$ Department of Mathematics, Govt. Motilal Vigyan Mahavidyalaya \\ Jehangirabad Road, Bhopal, M.P., India. E-mail: shilpijainbpl@gmail.com
}

Received 24 March 2017; accepted 22 April 2017

Abstract. In this paper, we prove a common fixed point theorem for occasionally weakly compatible mappings in modified intuitionistic fuzzy metric space. Consequently, our result improves and sharpens many known common fixed point theorems available in the existing literature of metric fixed point theory.

Keywords: Modified intuitionistic fuzzy metric space, common fixed point, fuzzy metric space, $t$-representable norms, occasionally weakly compatible mappings.

\section{AMS Mathematics Subject Classification (2010): 47H10, 54E35}

\section{Introduction}

The concept of fuzzy set was introduced in 1965 by Zadeh [22]. Since then, with a view to utilize this concept in topology and analysis, many authors have extensively developed by Park [15] which is now known as intuitionistic fuzzy metric space wherein notions of continuous $t$-norm and continuous $t$ - conorm are employed. Fixed point theory is one of the most fruitful and effective tools in mathematics which has enormous applications in several branches of science especially in chaos theory, game theory, theory of differential equation, etc. Intuitionistic fuzzy metric notion is also useful in modeling some physical problems wherein it is necessary to study the relationship between two probability functions as noticed by Gregori et al. [13]. For instance, it has a concrete physical visualization in the context of two slit experiment as the foundation of $E$-infinity theory of high energy physics whose details are available in El Naschie in [7, 8, 9]. As noticed by Gregori et al. [13], the topology induced by intuitionistic fuzzy metric coincides with the topology induced by fuzzy metric, Saadati et al. [20] reframed the idea of intuitionistic fuzzy metric spaces and proposed a new notion under the name 
Syed Shahnawaz Ali, Jainendra Jain, P.L. Sanodiya and Shilpi Jain

of modified intuitionistic fuzzy metric spaces by introducing the of continuous $t$-representable.

In this paper, we prove a common fixed point theorem for occasionally weakly compatible mappings in modified intuitionistic fuzzy metric space. Consequently result improves and sharpens many known common fixed point theorems available in the existing literature of metric fixed point theory.

\section{Preliminaries}

Definition 2.1. A binary operation $*:[0,1] \times[0,1] \rightarrow[0,1]$ is a continuous $t-$ norm if * satisfies following conditions:

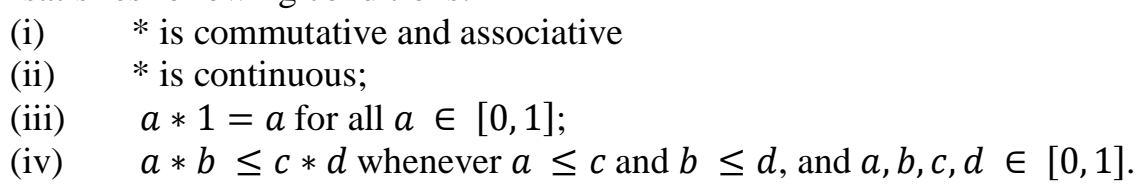

Definition 2.2. [9] A binary operation $\diamond:[0,1] \times[0,1] \rightarrow[0,1]$ is a continuous $t$-norm if 0 is satisfying the following conditions

(a) $\Delta$ is commutative and associative;

(b) $\diamond$ is continuous;

(c) $a \diamond 0=a$ for all $a \in[0,1]$;

(d) $a \diamond b \leq c \diamond d$ whenever $a \leq c$ and $b \leq d$, for all $a, b, c, d \in[0,1]$.

Lemma 2.1. [9] Consider the set $\mathrm{L}^{*}$ and operation $\leq_{\mathrm{L}^{*}}$ defined by:

$$
\begin{gathered}
L^{*}=\left\{\left(x_{1}, x_{2}\right):\left(x_{1}, x_{2}\right) \in[0,1]^{2} \text { and } x_{1}+x_{2} \leq 1\right\}, \\
\left(\mathrm{x}_{1}, \mathrm{x}_{2}\right) \leq_{L^{*}}\left(\mathrm{y}_{1}, \mathrm{y}_{2}\right) \Leftrightarrow \mathrm{x}_{1} \leq \mathrm{y}_{1}
\end{gathered}
$$

And $\mathrm{x}_{2} \geq \mathrm{y}_{2}$, for every $\left(x_{1}, x_{2}\right),\left(\mathrm{y}_{1}, \mathrm{y}_{2}\right) \in L^{*}$.

then $\left(L^{*}, L_{L^{*}}\right)$ is a complete lattice.

Definition 2.3. [1] An intuitionistic fuzzy set $\mathcal{A}_{\zeta, \eta}$ in a universe $U$ is an object $\mathcal{A}_{\zeta, \eta}=$ $\left\{\left(\zeta_{\mathcal{A}}(u), \eta_{\mathcal{A}}(u)\right) \mid u \in U\right\}$, where, for all $u \in U, \zeta_{\mathcal{A}}(u) \in[0,1]$ and $\eta_{\mathcal{A}}(u) \in[0,1]$ are called the membership degree and the non-membership degree, respectively of $u$ in $\mathcal{A}_{\zeta, \eta}$ and furthermore they satisfy $\zeta_{\mathcal{A}}(u)+\eta_{\mathcal{A}}(u) \leq 1$.

Definition 2.4. For every $z_{\alpha}=\left(x_{\alpha}, y_{\alpha}\right) \in L^{*}$ we define

$$
\mathrm{V}\left(z_{\alpha}\right)=\left(\sup \left(x_{\alpha}\right), \inf \left(y_{\alpha}\right)\right) \text {. }
$$

Since $z_{\alpha} \in L^{*}$ then $x_{\alpha}+y_{\alpha} \leq 1$ so $\sup \left(x_{\alpha}\right)+\inf \left(y_{\alpha}\right) \leq \sup \left(x_{\alpha}+y_{\alpha}\right) \leq 1$, that is $\vee\left(z_{\alpha}\right) \in L^{*}$. We denote its units by $0_{L^{*}}=(0,1)$ and $1_{L^{*}}=(1,0)$.

Classically, a triangular norm $*=T$ on $([0,1], \leq)$ is define as an increasing, commutative, associative mapping $T:[0,1]^{2} \rightarrow[0,1]$ satisfying $T(1, x)=1 * x=x$, for all $x \in[0,1]$. A triangular conorm $S=\emptyset$ is defined as an increasing, commutative, associative mapping $S:[0,1]^{2} \rightarrow[0,1]$ satisfying $S(0, x)=0 \diamond x=x$, for all $x \in$ $[0,1]$. Using the lattice $\left(L^{*}, \leq_{L^{*}}\right)$ these definitions can be straightforwardly extended.

Definition 2.5. $[9,14]$ A triangular norm $\left(t\right.$-norm) on $L^{*}$ is a mapping $\mathcal{T}:\left(L^{*}\right)^{2} \rightarrow L^{*}$ satisfying the following conditions: 
Fixed Point Theorem for Occasionally Weakly Compatible Mappings in Modified Intuitionistic Fuzzy Metric Space

1) $\left(\forall x \in L^{*}\right)\left(\mathcal{T}\left(x, 1_{L^{*}}\right)=x\right)$, (boundary condition)

2) $\left(\forall(x, y) \in\left(L^{*}\right)^{2}\right)(\mathcal{T}(x, y)=\mathcal{T}(y, x))$, (commutativity)

3) $\left(\forall(x, y, z) \in\left(L^{*}\right)^{3}\right)(\mathcal{T}(x, \mathcal{T}(y, z))=\mathcal{T}(\mathcal{T}(x, y), z))$, (associativity),

4) $\left(\forall\left(x, x^{\prime}, y, y^{\prime}\right) \in\left(L^{*}\right)^{4}\right)\left(x \leq_{L^{*}} x^{\prime}\right)$ and $\left(y \leq_{L^{*}} y^{\prime} \Rightarrow \mathcal{T}(x, y) \leq_{L^{*}} \mathcal{T}\left(x^{\prime}, y^{\prime}\right)\right)$. (monotonicity).

Definition 2.6. $[9,14]$ A continuous $t$-norm $\mathcal{T}$ on $L^{*}$ is called continuous

$t$-representable if and only if there exist a continuous $t$-norm * and a continuous

$t$-conorm $\varphi$ on $[0,1]$ such that, for all $x=\left(x_{1}, x_{2}\right), y=\left(y_{1}, y_{2}\right) \in L^{*}$,

$$
\mathcal{T}(x, y)=\left(x_{1} * y_{1}, x_{2} \diamond y_{2}\right) \text {. }
$$

Now define a sequence $\mathcal{T}^{n}$ recursively by $\mathcal{T}^{1}=\mathcal{T}$ and

$$
\mathcal{T}^{n}\left(x^{(1)}, \cdots, x^{(n+1)}\right)=\mathcal{T}\left(\mathcal{T}^{n-1}\left(x^{(1)}, \cdots, x^{(n)}\right), x^{(n+1)}\right)
$$

For $n \geq 2$ and $x^{(i)} \in L^{*}$.

We say the continuous $t$-representable norm is natural and write $\mathcal{T}_{n}$ whenever $\mathcal{T}_{n}(a, b)=\mathcal{T}_{n}(c, d)$ and $a \leq_{L^{*}} c$ implies $b \leq_{L^{*}} d$.

Definition 2.7. A negator on $L^{*}$ is any decreasing mapping $\mathcal{N}: L^{*} \rightarrow L^{*}$ satisfying $\mathcal{N}\left(0_{L^{*}}\right)=1_{L^{*}}$ and $\mathcal{N}\left(1_{L^{*}}\right)=0_{L^{*}}$. If $\mathcal{N}(\mathcal{N}(x))=x$, for all $x \in L^{*}$, then $\mathcal{N}$ is called an involutive negator. A negator on $[0,1]$ is a decreasing mapping $N:[0,1] \rightarrow[0,1]$ satisfying $N(0)=1$ and $N(1)=0 . N_{S}$ denotes the standard negator on $[0,1]$ defined as $N_{S}(x)=1-x$ for all $x \in[0,1]$.

Definition 2.8. Let $M, N$ are fuzzy sets from $X^{2} \times(0,+\infty)$ to $[0,1]$ such that $M(x, y, t)+N(x, y, t) \leq 1$ for all $x, y \in X$ and $t>0$. The $3-$ tuple $\left(X, \mathcal{M}_{M, N}, \mathcal{T}\right)$ is said to be a Modified Intuitionistic fuzzy metric space if $X$ is an arbitrary (non-empty) set, $\mathcal{T}$ is a continuous $t$-representable norm and $\mathcal{M}_{M, N}$ is a mapping $X^{2} \times(0,+\infty) \rightarrow$ $L^{*}$ (an intuitionistic fuzzy set, see Definition 3) satisfying the following conditions for every $x, y \in X$ and $t, s>0$ :

1) $\mathcal{M}_{M, N}(x, y, t)>_{L^{*}} 0_{L^{*}}$;

2) $\mathcal{M}_{M, N}(x, y, t)=1_{L^{*}}$ if and only if $x=y$;

3) $\mathcal{M}_{M, N}(x, y, t)=\mathcal{M}_{M, N}(y, x, t)$;

4) $\mathcal{M}_{M, N}(x, y, t+s) \geq_{L^{*}} \mathcal{T}\left(\mathcal{M}_{M, N}(x, z, t), \mathcal{M}_{M, N}(z, y, s)\right)$;

5) $\mathcal{M}_{M, N}(x, y, \cdot):(0, \infty) \rightarrow L^{*}$ is continuous.

In this case $\mathcal{M}_{M, N}$ is called an intuitionistic fuzzy metric space. Here, $\mathcal{M}_{M, N}(x, y, t)=(M(x, y, t), N(x, y, t))$.

In the sequel, we will call $\left(X, \mathcal{M}_{M, N}, \mathcal{T}\right)$ to be just a Modified Intuitionistic fuzzy metric space.

Remark 2.1. [18] In a Modified Intuitionistic fuzzy metric space $\left(X, \mathcal{M}_{M, N}, \mathcal{T}\right), M(x, y,$.$) is non decreasing and N(x, y,$.$) is non increasing for all$ $x, y \in X$. Hence $\mathcal{M}_{M, N}(x, y, t)$ is non decreasing with respect to $t$ for all $x, y \in X$. 
Syed Shahnawaz Ali, Jainendra Jain, P.L. Sanodiya and Shilpi Jain

Example 2.1. [11] Let $(X, d)$ be a metric space.

Define $\mathcal{T}(a, b)=\left\{a_{1} b_{1}, \min \left(a_{2}+b_{2}, 1\right)\right\}$ for all $a=\left(a_{1}, a_{2}\right)$ and $b=\left(b_{1}, b_{2}\right) \in L^{*}$ and let $M$ and $N$ be fuzzy sets on $X^{2} \times(0, \infty)$ defined as follows:

$$
\mathcal{M}_{M, N}(x, y, t)=(M(x, y, t), N(x, y, t))=\left(\frac{h t^{n}}{h t^{n}+m d(x, y)}, \frac{m d(x, y)}{h t^{n}+m d(x, y)}\right),
$$

for all $h, m, n, t \in \mathbb{R}^{+}$. Then $\left(X, \mathcal{M}_{M, N}, \mathcal{T}\right)$ is a modified intuitionistic fuzzy metric space.

Example 2.2. [1] Let $X=N$.

Define $\mathcal{T}(a, b)=\left\{\left(\max \left(0, a_{1}+b_{1}-1\right), a_{2}+b_{2}-a_{2} b_{2}\right\}\right.$ for all $a=\left(a_{1}, a_{2}\right)$ and $b=\left(b_{1}, b_{2}\right) \in L^{*}$ and let $M$ and $N$ be fuzzy sets on $X^{2} \times(0, \infty)$ defined as follows:

$$
\mathcal{M}_{M, N}(x, y, t)=(M(x, y, t), N(x, y, t))=\left\{\begin{array}{l}
\left(\frac{x}{y}, \frac{y-x}{y}\right) \text { if } x \leq y \\
\left(\frac{y}{x}, \frac{x-y}{x}\right) \text { if } y \leq x
\end{array}\right.
$$

for all $x, y \in X$ and $t>0$. Then $\left(X, \mathcal{M}_{M, N}, \mathcal{T}\right)$ is a modified intuitionistic fuzzy metric space.

Definition 2.9. [11] Let $\left(X, \mathcal{M}_{M, N}, \mathcal{T}\right)$ be a modified intuitionistic fuzzy metric space and $\left\{x_{n}\right\}$ be a sequence in $X$.

1. A sequence $\left\{x_{n}\right\}$ is said to be convergent to $x \in X$ in the modified intuitionistic fuzzy metric space $\left(X, \mathcal{M}_{M, N}, \mathcal{T}\right)$ and denoted by $x_{n} \stackrel{\mathcal{M}_{M, N}}{\longrightarrow} x$ if $\mathcal{M}_{M, N}\left(x_{n}, x, t\right) \rightarrow 1_{L^{*}}$ as $n \rightarrow \infty$ for every $t>0$.

2. A sequence $\left\{x_{n}\right\}$ in a modified intuitionistic fuzzy metric space $\left(X, \mathcal{M}_{M, N}, \mathcal{T}\right)$ is called a Cauchy sequence if for each $0<\varepsilon<1$ and $t>0$, there exists $n_{0} \in \mathbb{N}$ such that $\mathcal{M}_{M, N}\left(x_{n}, y_{m}, t\right)>_{L^{*}}\left(N_{s}(\varepsilon), \varepsilon\right)$, and for each $n, m \geq n_{0}$; here $N_{s}$ is the standard negator.

3. A modified intuitionistic fuzzy metric space is said to be complete if and only if every Cauchy sequence in this space is convergent. Henceforth, we assume that $\mathcal{T}$ is

a continuous $t$-norm on the lattice $\mathcal{L}$ such that for every $\mu \in L^{*} \backslash\left\{0_{L^{*}}, 1_{L^{*}}\right\}$, there exists $\lambda \in L^{*} \backslash\left\{0_{L^{*}}, 1_{L^{*}}\right\}$ such that $\mathcal{T}^{n-1}(\mathcal{N}(\lambda), \cdots, \mathcal{N}(\lambda)) \geq_{L^{*}} \mathcal{N}(\mu)$.

Lemma 2.2. [18] Let $\mathcal{M}_{M, N}$ be a modified intuitionistic fuzzy metric. Then for any $t>0$, $\mathcal{M}_{M, N}(x, y, t)$ is nondecreasing with respect to $t$ in $\left(L^{*}, \leq_{L^{*}}\right)$ for all $x, y \in X$.

Lemma 2.3. [3] Let $\left(X, \mathcal{M}_{M, N}, \mathcal{T}\right)$ be a modified intuitionistic fuzzy metric space and define $E_{\lambda, \mathcal{M}_{M, N}}: X^{2} \rightarrow \mathbb{R}^{+} \cup\{0\}$ by

$$
E_{\lambda, \mathcal{M}_{M, N}}(x, y)=\inf \left\{t>0: \mathcal{M}_{M, N}(x, y, t)>_{L^{*}} \mathcal{N}(\lambda)\right\}
$$

for each $\lambda \in L^{*} \backslash\left\{0_{L^{*}}, 1_{L^{*}}\right\}$ and $x, y \in X$ here, $\mathcal{N}$ is an involutive negator. Then we have (i) For any $\mu \in L^{*} \backslash\left\{0_{L^{*}}, 1_{L^{*}}\right\}$, there exists $\lambda \in L^{*} \backslash\left\{0_{L^{*}}, 1_{L^{*}}\right\}$ such that 
Fixed Point Theorem for Occasionally Weakly Compatible Mappings in Modified Intuitionistic Fuzzy Metric Space

$E_{\mu, \mathcal{M}_{M, N}}\left(x_{1}, x_{n}\right) \leq E_{\lambda, \mathcal{M}_{M, N}}\left(x_{1}, x_{2}\right)+E_{\lambda, \mathcal{M}_{M, N}}\left(x_{2}, x_{3}\right)+\cdots+E_{\lambda, \mathcal{M}_{M, N}}\left(x_{n-1}, x_{n}\right)$ for any $x_{1}, x_{2}, x_{3}, \cdots x_{n} \in X$.

(ii) The sequence $\left\{x_{n}\right\}_{n \in \mathbb{N}}$ in $X$ is convergent to $x$ with respect to modified intuitionistic fuzzy metric $\mathcal{M}_{M, N}$ if and only if $E_{\lambda, \mathcal{M}_{M, N}}\left(x_{n}, x\right) \rightarrow 0$. Also the sequence $\left\{x_{n}\right\}$ is a Cauchy sequence with respect to modified intuitionistic fuzzy metric $\mathcal{M}_{M, N}$ if and only if it is a Cauchy sequence with $E_{\lambda, \mathcal{M}_{M, N}}$.

Proof: For ( $i$ ), by the continuity of $t$-norms, for every $\mu \in L^{*} \backslash\left\{0_{L^{*}}, 1_{L^{*}}\right\}$, we can find a $\lambda \in L^{*} \backslash\left\{0_{L^{*}}, 1_{L^{*}}\right\}$ such that $\mathcal{T}(\mathcal{N}(\lambda), \mathcal{N}(\lambda)) \geq_{L^{*}} \mathcal{N}(\mu)$. By Definition 2.8 (iv) we have

$$
\begin{gathered}
\mathcal{M}_{M, N}\left(x, y, E_{\lambda, \mathcal{M}_{M, N}}\left(x_{1}, x_{2}\right)+E_{\lambda, \mathcal{M}_{M, N}}\left(x_{2}, x_{3}\right)+\cdots+E_{\lambda, \mathcal{M}_{M, N}}\left(x_{n-2}, x_{n-1}\right)\right. \\
\left.\quad+E_{\lambda, \mathcal{M}_{M, N}}\left(x_{n-1}, x_{n}\right)+n \delta\right) \\
\geq_{L^{*}} \mathcal{J}\left(\mathcal { M } _ { M , N } \left(x, z, E_{\lambda, \mathcal{M}_{M, N}}\left(x_{1}, x_{2}\right)+E_{\lambda, \mathcal{M}_{M, N}}\left(x_{2}, x_{3}\right)+\cdots+E_{\lambda, \mathcal{M}_{M, N}}\left(x_{n-3}, x_{n-2}\right)\right.\right. \\
\left.+\frac{n \delta}{2}\right), \mathcal{M}_{M, N}\left(z, y, E_{\lambda, \mathcal{M}_{M, N}}\left(x_{n-2}, x_{n-1}\right)+E_{\lambda, \mathcal{M}_{M, N}}\left(x_{n-1}, x_{n}\right)\right. \\
\left.\left.+\frac{n \delta}{2}\right)\right) \geq_{L^{*}} \mathcal{T}(\mathcal{N}(\lambda), \mathcal{N}(\lambda)) \geq_{L^{*}} \mathcal{N}(\mu)
\end{gathered}
$$

for every $\delta>0$, which implies that

$E_{\mu, \mathcal{M}_{M, N}}\left(x_{1}, x_{n}\right) \leq E_{\lambda, \mathcal{M}_{M, N}}\left(x_{1}, x_{2}\right)+E_{\lambda, \mathcal{M}_{M, N}}\left(x_{2}, x_{3}\right)+\cdots+E_{\lambda, \mathcal{M}_{M, N}}\left(x_{n-1}, x_{n}\right)+n \delta$

Since $\delta>0$ was arbitrary, we have

$$
E_{\mu, \mathcal{M}_{M, N}}\left(x_{1}, x_{n}\right) \leq E_{\lambda, \mathcal{M}_{M, N}}\left(x_{1}, x_{2}\right)+E_{\lambda, \mathcal{M}_{M, N}}\left(x_{2}, x_{3}\right)+\cdots+E_{\lambda, \mathcal{M}_{M, N}}\left(x_{n-1}, x_{n}\right) .
$$

For $(i i)$, we have $\mathcal{M}_{M, N}\left(x_{n}, x, \eta\right)>_{L^{*}} \mathcal{N}(\lambda) \Leftrightarrow E_{\lambda, \mathcal{M}_{M, N}}\left(x_{n}, x\right)<\eta$ for every $\eta>0$.

Lemma 2.4. Let $\left(X, \mathcal{M}_{M, N}, \mathcal{T}\right)$ be a modified intuitionistic fuzzy metric space. If $\mathcal{M}_{M, N}\left(x_{n}, x_{n+1}, t\right) \geq_{L^{*}} \mathcal{M}_{M, N}\left(x_{0}, x_{1}, \frac{t}{k^{n}}\right)$ for some $k<1$ and $n \in \mathbb{N}$ then $\left\{x_{n}\right\}$ is a Cauchy sequence.

Proof: For every $\lambda \in L \backslash\left\{0_{L^{*}}, 1_{L^{*}}\right\}$ and $x_{n} \in X$, we have

$$
\begin{aligned}
& E_{\lambda, \mathcal{M}_{M, N}}\left(x_{n}, x_{n+1}, t\right) \\
& =\inf \left\{t>0: \mathcal{M}_{M, N}\left(x_{n}, x_{n+1}, t\right)>_{L^{*}} \mathcal{N}(\lambda)\right\} \\
& \leq \inf \left\{t>0: \mathcal{M}_{M, N}\left(x_{0}, x_{1}, \frac{t}{k^{n}}\right)>_{L^{*}} \mathcal{N}(\lambda)\right\} \\
& =\inf \left\{k^{n} t: \mathcal{M}_{M, N}\left(x_{0}, x_{1}, t\right)>_{L} * \mathcal{N}(\lambda)\right\} \\
& =k^{n} \inf \left\{t>0: \mathcal{M}_{M, N}\left(x_{0}, x_{1}, t\right)>_{L^{*}} \mathcal{N}(\lambda)\right\} \\
& =k^{n} E_{\lambda, \mathcal{M}_{M, N}}\left(x_{n}, x_{n+1}, t\right)
\end{aligned}
$$

From lemma (2.3) for every $\mu \in L^{*} \backslash\left\{0_{L^{*}}, 1_{L^{*}}\right\}$ there exists $\lambda \in L^{*} \backslash\left\{0_{L^{*}}, 1_{L^{*}}\right\}$, such that $E_{\mu, \mathcal{M}_{M, N}}\left(x_{n}, x_{m}\right)$

$$
\leq E_{\lambda, \mathcal{M}_{M, N}}\left(x_{n}, x_{n+1}\right)+E_{\lambda, \mathcal{M}_{M, N}}\left(x_{n+1}, x_{n+2}\right)+\cdots+E_{\lambda, \mathcal{M}_{M, N}}\left(x_{m-1}, x_{m}\right)
$$

$\leq k^{n} E_{\lambda, \mathcal{M}_{M, N}}\left(x_{0}, x_{1}\right)+k^{n+1} E_{\lambda, \mathcal{M}_{M, N}}\left(x_{0}, x_{1}\right)+\cdots+k^{m-1} E_{\gamma, \mathcal{M}_{M, N}}\left(x_{0}, x_{1}\right)$

$=E_{\lambda, \mathcal{M}_{M, N}}\left(x_{0}, x_{1}\right) \sum_{j=n}^{m-1} k^{j} \rightarrow 0$.

Hence sequence $\left\{x_{n}\right\}$ is a Cauchy sequence. 
Syed Shahnawaz Ali, Jainendra Jain, P.L. Sanodiya and Shilpi Jain

Definition 2.10. Let $\left(X, \mathcal{M}_{M, N}, \mathcal{T}\right)$ be a modified intuitionistic fuzzy metric space. $\mathcal{M}$ is said to be continuous on $X \times X \times] 0, \infty[$ if

$$
\lim _{n \rightarrow \infty} \mathcal{M}_{M, N}\left(x_{n}, y_{n}, t_{n}\right)=\mathcal{M}_{M, N}(x, y, t),
$$

Whenever $\left\{\left(x_{n}, y_{n}, t_{n}\right)\right\}$ is a sequence in $\left.X \times X \times\right] 0, \infty[$ which converges to a point $(x, y, t) \in X \times X \times] 0, \infty[$ that is

$$
\begin{aligned}
& \lim _{n \rightarrow \infty} \mathcal{M}_{M, N}\left(x_{n}, x, t\right)=\lim _{n \rightarrow \infty} \mathcal{M}_{M, N}\left(y_{n}, y, t\right)=1_{L^{*}} \\
& \text { and } \lim _{n \rightarrow \infty} \mathcal{M}_{M, N}\left(x, y, t_{n}\right)=\mathcal{M}_{M, N}(x, y, t) .
\end{aligned}
$$

Lemma 2.5. Let $\left(X, \mathcal{M}_{M, N}, \mathcal{T}\right)$ be a modified intuitionistic fuzzy metric space and for all $x, y \in X, t>0$ and if for a number $k \in(0,1), \mathcal{M}_{M, N}(x, y, k t) \geq_{L^{*}} \mathcal{M}_{M, N}(x, y, t)$. Then $x=y$

Proof: For every $\lambda \in L \backslash\left\{0_{L^{*}}, 1_{L^{*}}\right\}$ and $x, y \in X$, we have

$E_{\lambda, \mathcal{M}_{M, N}}(x, y, t)$

$=\inf \left\{t>0: \mathcal{M}_{M, N}(x, y, t)>_{L} * \mathcal{N}(\lambda)\right\}$

$\leq \inf \left\{t>0: \mathcal{M}_{M, N}\left(x, y, \frac{t}{k}\right)>_{L} * \mathcal{N}(\lambda)\right\}$

$=\inf \left\{k t: \mathcal{M}_{M, N}(x, y, t)>_{L} * \mathcal{N}(\lambda)\right\}$

$=k \inf \left\{t>0: \mathcal{M}_{M, N}(x, y, t)>_{L} * \mathcal{N}(\lambda)\right\}$

$=k E_{\lambda, \mathcal{M}_{M, N}}(x, y, t)$

Therefore $E_{\lambda, \mathcal{M}_{M, N}}(x, y, t)=0$. Hence $x=y$.

Definition 2.11. Let $f$ and $g$ be mappings from a modified intuitionistic fuzzy metric space $\left(X, \mathcal{M}_{M, N}, \mathcal{T}\right)$ into itself. The maps $f$ and $g$ are said to be weakly commuting if $\mathcal{M}_{M, N}(A S z, S A z, t) \geq_{L^{*}} \mathcal{M}_{M, N}(A z, S z, t)$ for all $z \in X, t<0$.

Definition 2.12. A pair of self mappings $(f, g)$ of modified intuitionistic fuzzy metric space $\left(X, \mathcal{M}_{M, N}, \mathcal{T}\right)$ is said to be compatible if

$$
\lim _{n \rightarrow \infty} \mathcal{M}_{M, N}\left(f g x_{n}, g f x_{n}, t\right)=1_{L^{*}}
$$

whenever $\left\{x_{n}\right\}$ is a sequence in $X$ such that $\lim _{n \rightarrow \infty} f x_{n}=\lim _{n \rightarrow \infty}=g x_{n}=z$ for some $z \in X$.

Definition 2.13. Two self mappings $f$ and $g$ are called non compatible if there exist at least one sequence $\left\{x_{n}\right\}$ such that $\lim _{n \rightarrow \infty} f x_{n}=\lim _{n \rightarrow \infty}=g x_{n}=z$ for some $z \in X$ but either $\lim _{n \rightarrow \infty} \mathcal{M}_{M, N}\left(f g x_{n}, g f x_{n}, t\right) \neq 1_{L^{*}}$ or the limit does not exist for all $z \in X$.

Definition 2.14. A pair of self mappings $(f, g)$ of modified intuitionistic fuzzy metric space $\left(X, \mathcal{M}_{M, N}, \mathcal{T}\right)$ is said to be weakly compatible if they commute at coincidence point that is if $f u=g u$ for some $u \in X$, then $f g u=g f u$.

Definition 2.15. A pair of self mappings $(f, g)$ of modified intuitionistic fuzzy metric space $\left(X, \mathcal{M}_{M, N}, \mathcal{T}\right)$ is said to be occasionally weakly compatible if the pair $(f, g)$ 
Fixed Point Theorem for Occasionally Weakly Compatible Mappings in Modified Intuitionistic Fuzzy Metric Space

commutes at least one coincidence point that is there exists at least one point $x \in X$, such that $f x=g x$ and $f g x=g f x$.

Lemma 2.6.: Let $X$ be a set, $f$ and $g$ be occasionally weakly compatible self maps on $X$ of a modified intuitionistic fuzzy metric space $\left(X, \mathcal{M}_{M, N}, \mathcal{T}\right)$. If $f$ and $g$ have unique point of coincidence that is $w=f x=g x$ for $x \in X$, then $w$ is the unique common fixed point of $f$ and $g$.

\section{The main results}

Theorem 3.1. Let $\left(X, \mathcal{M}_{M, N}, \mathcal{T}\right)$ be a complete modified intuitionistic fuzzy metric space and let $A, B, S, T, P$ and $Q$ be self mappings of $X$. Let the pairs $(P, A B)$ and $(Q, S T)$ be occasionally weakly compatible. If there exist $k \in(0,1)$ such that $\mathcal{M}_{M, N}(P x, Q y, k t)$

$\geq_{L^{*}} \min \left\{\begin{array}{c}\mathcal{M}_{M, N}(A B x, S T y, t), \mathcal{M}_{M, N}(Q y, A B x, t), \mathcal{M}_{M, N}(P x, S T y, t), \mathcal{M}_{M, N}(A B x, P x, t), \\ \frac{a \mathcal{M}_{M, N}(P x, Q y, t)+b \mathcal{M}_{M, N}(P x, S T y, t)}{a \mathcal{M}_{M, N}(A B x, Q y, t)+b \mathcal{M}_{M, N}(A B x, S T y, t)}, \\ \frac{c \mathcal{M}_{M, N}(A B x, Q y, t)+d \mathcal{M}_{M, N}(A B x, S T y, t)}{c \mathcal{M}_{M, N}(Q y, S T y, t)+d}, \\ \frac{e \mathcal{M}_{M, N}(A B x, P x, t)+f \mathcal{M}_{M, N}(Q y, S T y, t)}{e+f}, \\ \frac{a \mathcal{M}_{M, N}(P x, S T y, t)+b \mathcal{M}_{M, N}(A B x, Q y, t)+c \mathcal{M}_{M, N}(A B x, S T y, t)}{a+b+c}\end{array}\right\}$

For all $x, y \in X$ all $t>0$ and $a, b, c, d, e, f \geq 0$ with $a \& b, c \& d$ and $e \& f$ cannot be simultaneously 0 , then there exist a unique point $w \in X$ such that $P w=A B w=w$ and a unique point $z \in X$ such that $Q z=S T z=z$. Moreover $w=z$, so that there is a unique common fixed point of $A, B, S, T, P$ and $Q$.

Proof: Let the pairs $(P, A B)$ and $(Q, S T)$ be occasionally weakly compatible, so there are points $x, y \in X$ such that $P x=A B x$ and $Q y=S T y$. We claim $P x=Q y$. If not, by inequality (1)

$\mathcal{M}_{M, N}(P x, Q y, k t)$

$$
\geq_{L^{*}} \min \left\{\begin{array}{c}
\mathcal{M}_{M, N}(A B x, S T y, t), \mathcal{M}_{M, N}(Q y, A B x, t), \mathcal{M}_{M, N}(P x, S T y, t), \mathcal{M}_{M, N}(A B x, P x, t), \\
\frac{a \mathcal{M}_{M, N}(P x, Q y, t)+b \mathcal{M}_{M, N}(P x, S T y, t)}{a \mathcal{M}_{M, N}(A B x, Q y, t)+b \mathcal{M}_{M, N}(A B x, S T y, t)}, \\
\frac{c \mathcal{M}_{M, N}(A B x, Q y, t)+d \mathcal{M}_{M, N}(A B x, S T y, t)}{c \mathcal{M}_{M, N}(Q y, S T y, t)+d}, \\
\frac{e \mathcal{M}_{M, N}(A B x, P x, t)+f \mathcal{M}_{M, N}(Q y, S T y, t)}{e+f}, \\
\frac{a \mathcal{M}_{M, N}(P x, S T y, t)+b \mathcal{M}_{M, N}(A B x, Q y, t)+c \mathcal{M}_{M, N}(A B x, S T y, t)}{a+b+c}
\end{array}\right\}
$$


Syed Shahnawaz Ali, Jainendra Jain, P.L. Sanodiya and Shilpi Jain

$$
=\text { L }_{L^{*}} \min \left\{\begin{array}{c}
\mathcal{M}_{M, N}(P x, Q y, t), \mathcal{M}_{M, N}(Q y, P x, t), \mathcal{M}_{M, N}(P x, Q y, t), \mathcal{M}_{M, N}(P x, P x, t), \\
\frac{a \mathcal{M}_{M, N}(P x, Q y, t)+b \mathcal{M}_{M, N}(P x, Q y, t)}{a \mathcal{M}_{M, N}(P x, Q y, t)+b \mathcal{M}_{M, N}(P x, Q y, t)}, \\
\frac{c \mathcal{M}_{M, N}(P x, Q y, t)+d \mathcal{M}_{M, N}(P x, Q y, t)}{c \mathcal{M}_{M, N}(Q y, Q y, t)+d}, \\
\frac{e \mathcal{M}_{M, N}(P x, P x, t)+f \mathcal{M}_{M, N}(Q y, Q y, t)}{e+f}, \\
\frac{a \mathcal{M}_{M, N}(P x, Q y, t)+b \mathcal{M}_{M, N}(P x, Q y, t)+c \mathcal{M}_{M, N}(P x, Q y, t)}{a+b+c}
\end{array}\right\}
$$

Therefore $P x=Q y$ that is $P x=A B x=Q y=S T y$. Suppose that there is another point $z$ such that $P z=A B z$ then by inequality (1) we have $P z=A B z=Q z=S T z$. So $P x=P z$ and $w=P x=A B x$ is the unique point of coincidence of $P$ and $A B$. By lemma 2.6, $w$ is the only common fixed point of $P$ and $A B$. Similarly there is a unique point $z \in X$ such that $z=Q z=S T z$.

Assume that $w \neq z$. We have, by inequality (1)

$$
\geq_{L^{*}} \min \left\{\begin{array}{c}
\mathcal{M}_{M, N}(w, z, k t)=\mathcal{M}_{M, N}(P w, Q z, k t) \geq_{L^{*}} \min \\
\mathcal{M}_{M, N}(w, z, t), \mathcal{M}_{M, N}(z, w, t), \mathcal{M}_{M, N}(w, z, t), \mathcal{M}_{M, N}(w, w, t), \\
\frac{a \mathcal{M}_{M, N}(w, z, t)+b \mathcal{M}_{M, N}(w, z, t)}{a \mathcal{M}_{M, N}(w, z, t)+b \mathcal{M}_{M, N}(w, z, t)}, \\
\frac{c \mathcal{M}_{M, N}(w, z, t)+d \mathcal{M}_{M, N}(w, z, t)}{c \mathcal{M}_{M, N}(z, z, t)+d}, \\
\frac{e \mathcal{M}_{M, N}(w, w, t)+f \mathcal{M}_{M, N}(z, z, t)}{e+f}, \\
\frac{a \mathcal{M}_{M, N}(w, z, t)+b \mathcal{M}_{M, N}(w, z, t)+c \mathcal{M}_{M, N}(w, z, t)}{a+b+c} \\
\min \left\{\begin{array}{c}
\mathcal{M}_{M, N}(w, z, t), \mathcal{M}_{M, N}(z, w, t), \mathcal{M}_{M, N}(w, z, t), 1_{L^{*},} \\
\mathcal{M}_{M, N}(w, z, t), \\
\mathcal{M}_{M, N}(w, z, t), \\
1_{L^{*}}, \\
\mathcal{M}_{M, N}(w, z, t) \\
{ }_{L^{*}} \mathcal{M}_{M, N}(w, z, t)
\end{array}\right\}
\end{array}\right\}
$$

Therefore we have $z=w$, by lemma $2.6, z$ is a common fixed point of $A, B, S, T, P$ and $Q$.

For uniqueness, let $u$ be another common fixed point of $A, B, S, T, P$ and $Q$. Then 
Fixed Point Theorem for Occasionally Weakly Compatible Mappings in Modified Intuitionistic Fuzzy Metric Space

$$
\begin{aligned}
& \mathcal{M}_{M, N}(z, u, k t)=\mathcal{M}_{M, N}(P z, Q u, k t) \\
& \geq_{L^{*}} \min \left\{\begin{array}{c}
\mathcal{M}_{M, N}(A B z, S T u, t), \mathcal{M}_{M, N}(Q u, A B z, t), \mathcal{M}_{M, N}(P z, S T u, t), \mathcal{M}_{M, N}(A B z, P z, t), \\
\frac{a \mathcal{M}_{M, N}(P z, Q u, t)+b \mathcal{M}_{M, N}(P z, S T u, t)}{a \mathcal{M}_{M, N}(A B z, Q u, t)+b \mathcal{M}_{M, N}(A B z, S T u, t)}, \\
\frac{c \mathcal{M}_{M, N}(A B z, Q u, t)+d \mathcal{M}_{M, N}(A B z, S T u, t)}{c \mathcal{M}_{M, N}(Q u, S T u, t)+d}, \\
\frac{e \mathcal{M}_{M, N}(A B z, P z, t)+f \mathcal{M}_{M, N}(Q u, S T u, t)}{e+f}, \\
\frac{a \mathcal{M}_{M, N}(P z, S T u, t)+b \mathcal{M}_{M, N}(A B z, Q u, t)+c \mathcal{M}_{M, N}(A B z, S T u, t)}{a+b+c}
\end{array}\right\} \\
& =_{L^{*}} \min \left\{\begin{array}{c}
\mathcal{M}_{M, N}(z, u, t), \mathcal{M}_{M, N}(u, z, t), \mathcal{M}_{M, N}(z, u, t), \mathcal{M}_{M, N}(z, z, t), \\
\frac{a \mathcal{M}_{M, N}(z, u, t)+b \mathcal{M}_{M, N}(z, u, t)}{a \mathcal{M}_{M, N}(z, u, t)+b \mathcal{M}_{M, N}(z, u, t)}, \\
\frac{c \mathcal{M}_{M, N}(z, u, t)+d \mathcal{M}_{M, N}(z, u, t)}{c \mathcal{M}_{M, N}(u, u, t)+d}, \\
\frac{e \mathcal{M}_{M, N}(z, z, t)+f \mathcal{M}_{M, N}(u, u, t)}{e+f}, \\
\frac{a \mathcal{M}_{M, N}(z, u, t)+b \mathcal{M}_{M, N}(z, u, t)+c \mathcal{M}_{M, N}(z, u, t)}{a+b+c}
\end{array}\right\} \\
& ={ }_{L^{*}} \min \left\{\begin{array}{c}
\mathcal{M}_{M, N}(z, u, t), \\
\mathcal{M}_{M, N}(u, z, t), \mathcal{M}_{M, N}(z, u, t), 1_{L^{*}} \\
\mathcal{M}_{M, N}(z, u, t), \\
\mathcal{M}_{M, N}(z, u, t), \\
1_{L^{*}}, \\
\mathcal{M}_{M, N}(z, u, t)
\end{array}\right\} \\
& ={ }_{L^{*}} \mathcal{M}_{M, N}(z, u, t)
\end{aligned}
$$

Therefore by lemma 2.6 we have $z=u$.

\section{Conclusions}

In this paper, we prove a common fixed point theorem for occasionally weakly compatible mappings in modified intuitionistic fuzzy metric space. Our result is more interesting and useful for other researchers.

\section{REFERENCES}

1. K.T.Atanassov, Intuitionistic fuzzy sets, Fuzzy Sets and Systems, 20 (1986) 87-96.

2. H.M.Abu-Donia and A.A.Nase, Common fixed point theorems intuitionistic fuzzy metric spaces, Fuzzy Systems and Mathematics, 22 (2008) 100-106.

3. H.Adibi, Y.J.Cho, D.O.Regan and R.Saadati, Common fixed point theorems in $\mathcal{L}$-fuzzy metric spaces, Appl. Math. Comp., 182 (2006) 820-828.

4. C.Alaca, D.Turkoglu and C.Yildiz, Fixed points in intuitionistic fuzzy metric spaces, Chaos, Solitons and Fractals, 29 (2006) 1073 -1078.

5. G.Deschrijver and E.E.Kerre, On the relationship between some extensions of fuzzy set theory, Fuzzy Sets and Systems, 23 (2003) 227-235. 
Syed Shahnawaz Ali, Jainendra Jain, P.L. Sanodiya and Shilpi Jain

6. G.Deschrijver, C.Corenelis and E.E.Kerre, On the representation of intuitionistic fuzzy $t$-norms and $t$-conorms, IEEE Transactions on Fuzzy Systems, 12 (2004) 4561.

7. M.S.El Naschie, A review of $E$-infinity theory and the mass spectrum of high energy particle physics, Chaos, Solitons and Fractals, 19 (2004) 209 - 236.

8. M.S.El Naschie, On two new fuzzy kahler manifolds, Klein Modular space and t Hooft Holographic principles, Chaos, Solitons and Fractals, 29 (2006) 876 - 881.

9. M.S.El Naschie, The idealized quantum two-slit gedanken experiment revisited criticism and reinterpretation, Chaos, Solitons and Fractals, 27 (2006) 843 - 849.

10. J.Goguen, $\mathcal{L}-$ fuzzy sets, Journal of Mathematical Analysis and Applications, 18 (1967) $145-174$.

11. A.George and P.Veeramani, On some results in fuzzy metric spaces, Fuzzy Sets and Systems, 64 (1994) 395-399.

12. M.Grabiec, Fixed points in fuzzy metric spaces, Fuzzy Sets and Systems, 27 (1988) $385-389$.

13. V.Gregori, S.Romaguera and P.A.Veereamani, Note on intuitionistic fuzzy metric spaces, Chaos, Solitons and Fractals, 28 (2006) $902-905$.

14. V.Gregori and S.Sapena, On fixed point theorems in fuzzy metric spaces, Fuzzy Sets and Systems, 125 (2002) 245-253.

15. J.H.Park, Intuitionistic fuzzy metric spaces, Chaos, Solitons and Fractals, 22 (2004) 1039-1046.

16. I.Kramosil and J.Michalek, Fuzzy metric and statistical metric spaces, Kybernetica, 11 (1975) 326-334.

17. R.P.Pant, Common fixed points of non commuting mappings, Journal of Mathematical Analysis and Applications, 188 (1994) 436 - 440.

18. R.Saadati and J.H.Park, On the intuitionistic fuzzy topological spaces, Chaos, Solitons and Fractals, 27 (2006) 331 -344.

19. R.Saadati, A.Razani and H.Adibi, A common fixed point theorem in $\mathcal{L}-$ fuzzy metric spaces, Chaos, Solitons and Fractals, 33 (2007) 358 -363.

20. R.Saadati, S.Sedgi and N.Shobe, Modified intuitionistic fuzzy metric spaces and some fixed point theorems, Chaos, Solitons and Fractals, 38 (2008) 36- 47.

21. S.Sharma, Common fixed point theorems in fuzzy metric spaces, Fuzzy sets and Systems, 127 (2002) 345-352.

22. L.A.Zadeh, Fuzzy sets, Inform and Control, 8 (1965) 338-353.

23. K.Jha, M.Imdad and U.Rajopadhyaya, Fixed point theorems for occasionally weakly compatible mappings in semi-metric space, Annals of Pure and Applied Mathematics, 5(2) (2014) 153-157.

24. A.Sugandhi, S.K.Tiwari and A.Pariya, Common fixed point theorems in intuitionistic fuzzy symmetric spaces for occasionally weakly compatible maps satisfying contractive condition of integral type, Annals of Pure and Applied Mathematics, 11(2) (2016) 11-19. 